\title{
Methodology for registration of distended rectums in pelvic CT studies
}

\author{
B. Rodriquez-Vila \\ F. Garcia-Vicente \\ E. J. Gomez
}

Purpose: Accurate delineation of the rectum is of high importance in off-line adaptive radiation therapy since it is a major dose-limiting organ in prostate cancer radiotherapy. The intensity-based deformable image registration (DIR) methods cannot create a correct spatial transformation if there is no correspondence between the template and the target images. The variation of rectal filling, gas, or feces, creates a noncorrespondence in image intensities that becomes a great obstacle for intensitybased DIR.

Methods: In this study the authors have designed and implemented a semiautomatic method to create a rectum mask in pelvic computed tomography (CT) images. The method, that includes a DIR based on the demons algorithm, has been tested in 13 prostate cancer cases, each comprising of two CT scans, for a total of 26 CT scans.

Results: The use of the manual segmentation in the planning image and the proposed rectum mask method (RMM) method in the daily image leads to an improvement in the DIR performance in pelvic CT images, obtaining a mean value of overlap volume index $=0.89$, close to the values obtained using the manual segmentations in both images.

Conclusions: The application of the RMM method in the daily image and the manual segmentations in the planning image during prostate cancer treatments increases the performance of the registration in presence of rectal fillings, obtaining very good agreement with a physician's manual contours.

\section{INTRODUCTION}

In modern radiation therapy (RT) the problem of organs' motion and deformation over the course of the treatment is becoming an important research area as the techniques for conformal therapy improve. ${ }^{1,2}$ These techniques offer important benefits since steeper gradients between the target volume and critical organs allow increasing the therapeutic dose to the tumor while reducing the radiation to the surrounding high-risk organs. The problem of these high gradients is that organ location and shape variations between treatment sessions become even more crucial ${ }^{2}$ and relatively little organ motion is required to bring parts of the tumor outside of the therapeutic region or to bring healthy critical tissues inside. ${ }^{3}$ Toward individualized RT, Yan et $a l^{4}$ introduced the concept of offline adaptive radiation therapy (OART), in which organs motion for an individual patient is measured over the course of treatment, and the subsequent delivery can be modified using a systematic feedback of the geometric and dosimetric information from previous fractions.
Anyhow, one of the limiting factors in the systematic feedback is the lack of accurate manual segmentations of the prostate and the OARTs. Manual segmentation of the daily images is time consuming, expensive, and not practical. Moreover, it introduces uncertainties associated with the inter-rater and intrarater variability. ${ }^{6}$ Another requirement in the OART feedback is to accurately account for anatomical variations.

Deformable image registration (DIR) techniques have proved to be effective in both the automatic segmentation and to account for such variations in organs that deform and move relative to one another. ${ }^{7}$ DIR between the planning computed tomography (CT) scan and the daily CT scan plays a key role in OART since it is the basis for other techniques such as dose accumulation, ${ }^{8}$ recontouring, ${ }^{9}$ and reoptimization. ${ }^{10}$ Daily images [obtained using image modalities as cone beam CT or megavoltage computed tomography (MVCT) images], associated dose reconstruction, and DIR methods can be used to compute the actual patient-specific dose distribution.

Different approaches have been proposed for nonrigid registration of serial CT images of the pelvic regions, and several 
of them are intensity-based DIR methods. ${ }^{11-14}$ However, in some situations physical one-to-one correspondence between images may not exist due to the insertion or removal of some image content. This inconsistency between two image studies could severely reduce the performance of an intensity-based DIR algorithm. ${ }^{5}$ In the case of prostate cancer patients, this inconsistency may be due to the presence or absence of bowel gas and solid feces (rectal fillings from this point forward) during the different sessions. ${ }^{14}$ The issue of noncorrespondence between a pair of prostate CT images with and without rectal gas is not well addressed in the current literature. ${ }^{7}$ Among the exceptions are two studies: while Foskey et al. ${ }^{5}$ tried to deflate the gassy region, forcing it to shrink to a virtual point, Gao et al. ${ }^{14}$ proposed the opposite, inflating an artificial gas bubble on the planning image. In both cases one of the images is supposed to have almost no rectal gas, while the other is gaseous. Nevertheless, only Gao et al. evaluate the quality of the proposed solution over the rectum.

In this study the authors propose a segmentation method for the rectum to mask its contents (gas and/or feces), in order to improve the performance of intensity-based DIR methods in pelvic CT scans which present distended rectums. The article is focused on obtaining the deformation field from the DIR, needed for the automatic recontouring as well as the computation of the actual patient-specific dose distribution and the dose accumulation. So, the final purpose is not developing a whole OART methodology, with several scans per patient during the treatment, but presenting an image-processing tool for a better DIR performance.

\section{MATERIAL AND METHODS}

\section{II.A. Patient data}

Images from 13 patients undergoing prostate cancer radiation therapy at University Hospital of La Princesa were used for this study. Each patient underwent two simulation CT scans for conformal RT (3D-CRT) planning purposes; the first CT scan of each patient was originally discarded by the physicist for planning purposes due to the presence of abnormal shape of the rectum (due to strangulations) or abnormal size of the bladder. Thus, 26 CT scans were obtained with a Toshiba Asteion CT (Toshiba Medical Systems, Japan). RT treatment plans were developed using a XIO 4.34.02 (Computerized Medical Systems, USA) treatment planning system.

The pelvic CT scans are composed of 40 slices of 512 $\times 512$ pixels, with a spatial resolution of $0.937 \times 0.937$ $\times 3 \mathrm{~mm}^{3}$, showing all the structures of interest in the pelvic area. The rectum, between the rectosigmoid flexure and the anus, the bladder, the prostate, the femoral heads, and the patient contour were manually delineated in the 26 CT studies. In some patients, gold fiducial markers were implanted in the prostate for localization purposes.

\section{II.B. Workflow description}

The proposed method is based on the hypothesis that the DIR algorithm performance will be better if the rectum is segmented in both the daily and the planning image. Since the manual delineation of the daily image is not usually available, the proposed method tries to create a rough mask of the rectum that should help during the registration process. Figure 1 illustrates the algorithm proposed, where processes are represented as ellipsoids and images are represented as rectangles. Basically, there are four main processes associated with this algorithm: edge-preserving smoothing, rigid alignment of bony structures, rectum masking, and DIR. The rectum masking process has been completely developed by the authors using MATLAB ${ }^{\circledR}$, while the other processes are wellknown algorithms available also in MATLAB.

\section{II.B.1. Edge-preserving smoothing}

CT images are formed by tomographic reconstruction and distorted, by definition, by several different artifacts. ${ }^{15}$ To smooth these differences without degrading image contrast resolution, an anisotropic diffusion filter has been applied to both planning and daily CT for noise reduction. ${ }^{16}$ This step should allow the registration between a planning CT image and a daily cone-beam CT image with poorer quality. Lu et $a .^{17}$ suggested a similar solution for the registration of kilovoltage computed tomography (kVCT) images and MVCT images, where the differences in the photon energy and data acquisition mechanism make MVCT images noisier and lower contrast than diagnostic kVCT. Nevertheless, the present study has not evaluated these possibilities and has been developed using only kVCT diagnostic scans.

\section{II.B.2. Rigid alignment}

Despite the efforts of clinicians and industry, the reproducibility of the patient setup during the treatment is still a problem. ${ }^{18}$ To overcome small setup displacements and initialize the DIR algorithm, a rigid registration method is applied. The bony structures are segmented by threshold segmentation and a rigid-body alignment algorithm is used. The obtained transformation is posteriorly applied to the daily CT image.

\section{II.B.3. Rectum mask method (RMM)}

The automatic delineation of the rectum wall in CT images is a great challenge due to its variability in shape and content. The rectum is mainly surrounded by conjunctive tissue, except in the regions where it is in contact with the prostate, the seminal vesicles, or the bladder, and near the anus, where it is surrounded by muscles. Therefore, a rough initial segmentation can be done using the histogram of the intensities of the CT image. Some regions can be identified studying the intensities (Fig. 2): background, rectal fillings, conjunctive-like tissue, muscular tissue, and bones.

The regions belonging to the foreground where intensity is lower than the conjunctive tissue are labeled as rectal fillings. The image used to guide the segmentation process will be the result of subtracting the internal fillings to the conjunctive tissue image [see the original CT slice in Fig. 4(a) and the transformed CT slice in Fig. 4(b)]. 


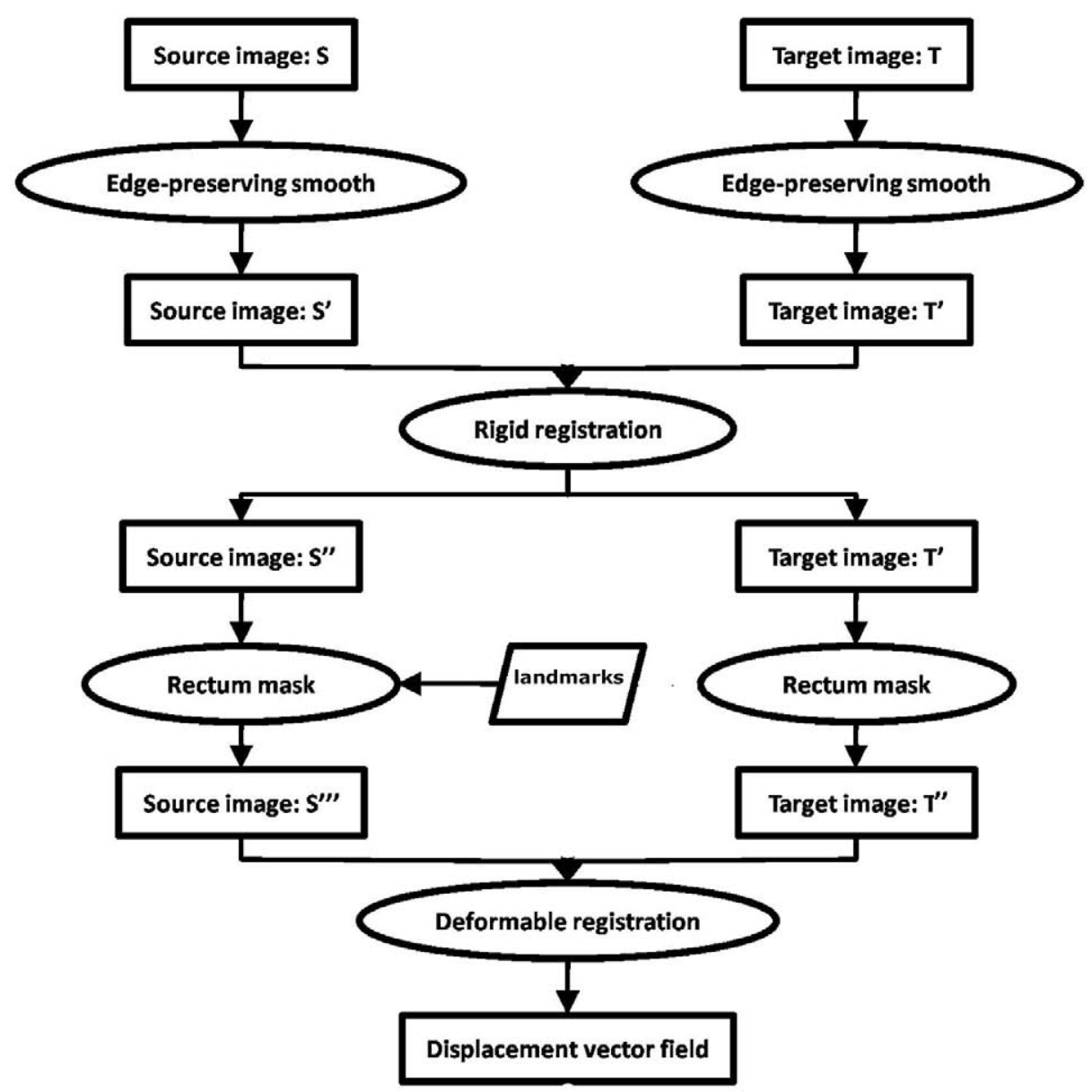

FIG. 1. Graphical representation of the proposed workflow for the registration of distended rectums.

The next step in the RMM method is the inclusion of shape information. The rectum between the rectosigmoid flexure and the anus can be considered as a slightly bent tube or cylinder with variable content. The surface of a cylinder can be represented as a flat surface using the information of cylindrical coordinates: the radius (in the $\mathrm{Z}$-axis) represented against the polar angle (X-axis) and the $\mathrm{Z}$ coordinate (Y-axis). A similar representation can be used in the case of the rectum, by first "straightening" it and representing the distance from the

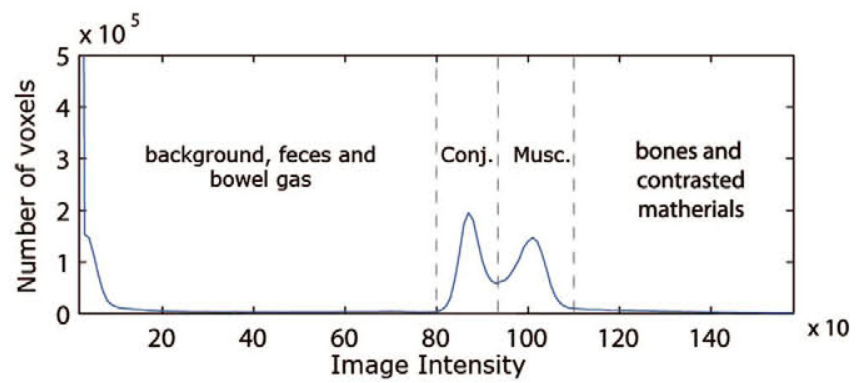

FIG. 2. Histogram of a pelvic CT: the peak around the minimum is formed by the image background; the second peak groups the intensities related to conjunctive-like tissue, while the nearby third peak groups pixels represent muscular-like tissues. Bony structures and contrasted tissue are represented by the higher intensities over the last peak, while rectal fillings appear between the background intensities and the conjunctive-like intensities. rectum wall to the rectum's centerline in a similar way as the radius of cylinder (see Fig. 3).

Following this scheme, a new 3D image is computed by transforming the preprocessed image. A polar-log transformation ${ }^{19}$ is applied to every slice of the preprocessed image using the rectum's center of mass as the kernel of the transformation [see Fig. 4(c)]. The final 3D image is constructed by sticking together all the transformed slices. The size of the transformed image will depend on the angular resolution used to scan the original image and the maximum search radius. In our case, an angular resolution of $1^{\circ}$ and a maximum radius of $8 \mathrm{~cm}$ (selected empirically, and encloses by far the largest rectum in our image set) have been selected. Main advantages of the polar-log transformation are that it allows increasing the spatial resolution of the selected region while reducing the size of the image, and that every isolated muscular structure in the original image appears as an isolated structure in the transformed image, except for the rectum [see Fig. 4(c)], delimitated by a smooth, almost horizontal surface, as the one represented in Fig. 3(c).

The center of mass of the rectum at each slice of the planning image is computed using the manually delineated contours. However, since the daily image should not have manually delineated contours, it requires the introduction of two landmark points by a specialist: one on the rectosigmoid flexure and the other on the anus. Both landmarks must be 


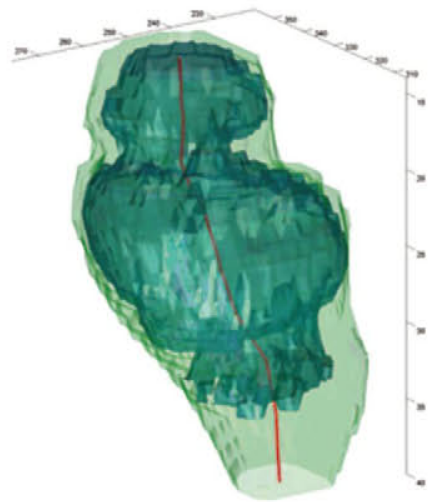

(a)

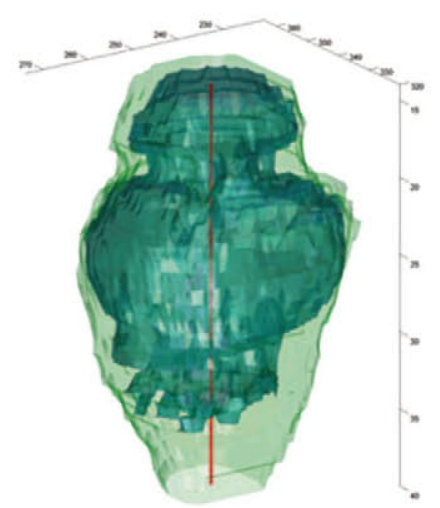

(b)

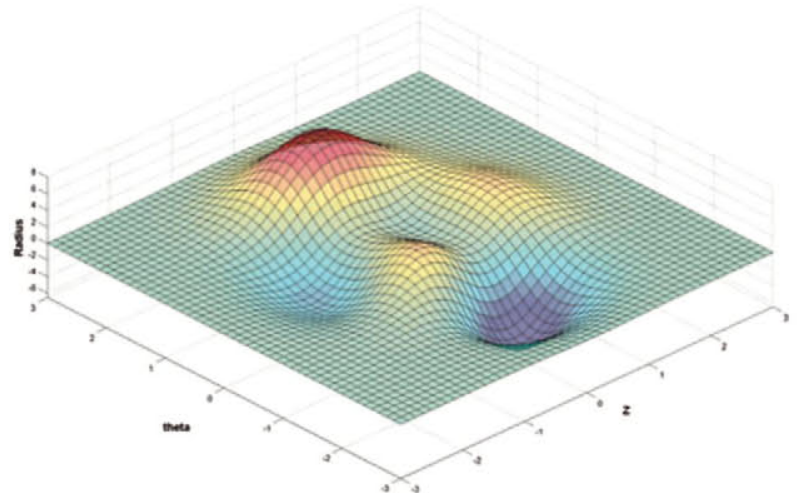

(c)

FIG. 3. (a) 3D reconstruction of the rectum and its internal fillings, showing the centerline of the rectum; (b) 3D reconstruction of the straightened rectum and its internal fillings, showing the straightened centerline; (c) Representation of the surface of the rectum wall using cylindrical coordinates: theta in the $\mathrm{X}$-axis, $\mathrm{Z}$ in the $\mathrm{Y}$-axis, and radius in the $\mathrm{Z}$-axis.

manually situated by selecting the closest pixel to the center of mass of the rectum on the correspondent slice.

The centerline calculation is automatically computed slice by slice, and from the rectosigmoid flexure to the anus, computing the center of mass of the rectum on a searching area of $5 \times 5 \mathrm{~cm}^{2}$, empirically determinated. The searching area is centered on a point computed using the centerline point in the previous slice and the known displacement between rectum slices in the planning image. The centerline point $\vec{p}$ is computed as the center of mass of the gas pixels $\vec{g}_{i}$ and the muscular pixels $\vec{m}_{i}$ included in the searching area:

$$
\vec{p}=\frac{1}{M+G}\left(\sum_{i=1}^{M} \vec{m}_{i}+\sum_{i=1}^{G} \vec{g}_{i}\right) .
$$

The robustness of the searching process decreases with the distance of the current slice to the manual landmark. Therefore, the second seed is also used to repeat the process from bottom to top. Gaussian weighting (centered in the seeds and

CT1
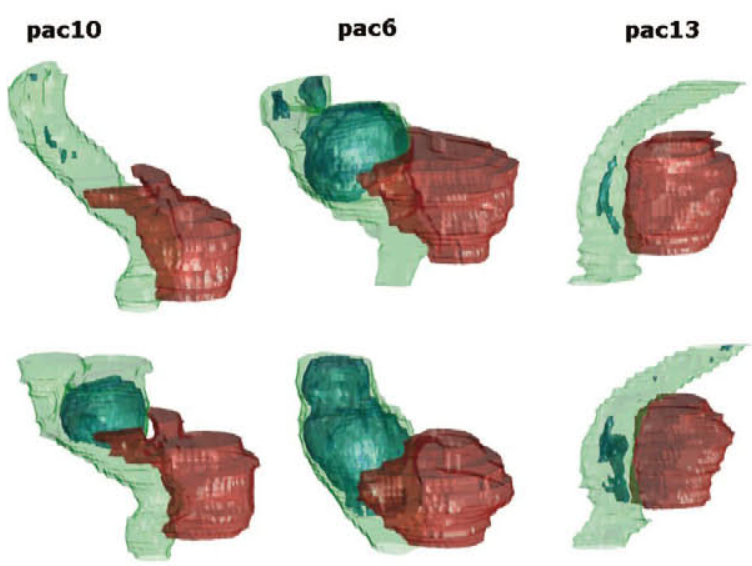

FIG. 4. 3D representations of the prostate, the rectum, and its internal fillings. Three different cases presented in the image database: (left) registration of an empty rectum with a rectum with big amount of internal fillings; (middle) registration of a rectum with big amount of internal fillings in both images; and (right) registration of a rectum with small amount of internal fillings in both images. with $\sigma=0.4 d$, $d$ being the distance between the seeds) is used to combine both estimations in a final centerline.

Finally, the rectum wall delineation is computed by iteratively deforming an initial mesh automatically created from the centerline. This initial mesh forms a horizontal surface 1 $\mathrm{cm}$ from the centerline of the rectum. The mesh is deformed applying external and internal forces. The external forces, computed from the intensity in the transformed image, are formed by two different components: (1) rectal filling pixels, which drive the mesh to higher radius (propagation forces) and (2) conjunctive-like pixels, which attract the mesh to their level (advection forces). The internal forces impose a smoothness condition (curvature forces).

\section{II.B.4. Deformable registration}

There are numerous intensity-based DIR methods in the literature, several of them on RT images of prostate cancer. We have chosen the Demons algorithm, ${ }^{20}$ developed in the Institut National de Recherche en Informatique et en Automatique (INRIA, France) because of its simplicity and its time performance. For clarity purposes, the planning $\mathrm{CT}$ is referred as the source image $\mathrm{S}(\vec{x})$, while the daily session image is referred as the target image $T(\overrightarrow{\mathrm{x}})$.

The functional of energy used in the implementation is formulated as

$$
E(\overrightarrow{\mathrm{x}})=(T(\overrightarrow{\mathrm{x}})-S(\overrightarrow{\mathrm{x}}+\overrightarrow{\mathrm{u}}(\overrightarrow{\mathrm{x}})))^{2} \cdot\left(1+\alpha^{2} \overrightarrow{\mathrm{u}}(\overrightarrow{\mathrm{x}})^{2}\right) .
$$

The displacement vector field $\overrightarrow{\mathrm{u}}(\overrightarrow{\mathrm{x}})$ is computed using gradient information from both images, obtaining a "symmetric" registration, such as the one performed by Wang: ${ }^{12}$

$$
\begin{aligned}
\overrightarrow{\mathrm{u}}(\overrightarrow{\mathrm{x}})= & \nabla \mathrm{E}(\overrightarrow{\mathrm{x}})=(T(\overrightarrow{\mathrm{x}})-S(\overrightarrow{\mathrm{x}}+\overrightarrow{\mathrm{u}}(\overrightarrow{\mathrm{x}}))) \\
& \cdot\left(\frac{\nabla T(\overrightarrow{\mathrm{x}})}{(\nabla T(\overrightarrow{\mathrm{x}}))^{2}+\alpha^{2}(T(\overrightarrow{\mathrm{x}})-S(\overrightarrow{\mathrm{x}}+\overrightarrow{\mathrm{u}}(\overrightarrow{\mathrm{x}})))^{2}}\right. \\
& \left.+\frac{\nabla S(\overrightarrow{\mathrm{x}}+\overrightarrow{\mathrm{u}}(\overrightarrow{\mathrm{x}}))}{(\nabla S(\overrightarrow{\mathrm{x}}+\overrightarrow{\mathrm{u}}(\overrightarrow{\mathrm{x}})))^{2}+\alpha^{2}(T(\overrightarrow{\mathrm{x}})-S(\overrightarrow{\mathrm{x}}+\overrightarrow{\mathrm{u}}(\overrightarrow{\mathrm{x}})))^{2}}\right) .
\end{aligned}
$$


On one hand, different fluid-like regularizations of the computed displacement are obtained by using Gaussian convolution with different kernels. On the other hand, the value of $\alpha$ allows modifying the weight of the regularization term (in terms of diffusion) in the energy functional. Gaussian filter of $\sigma=4$ and $15 \times 15 \times 15 \mathrm{~mm}^{3}$, and $\alpha=4$, and a multiresolution strategy in three levels were used during the experiments.

Inspired by the Juggle algorithm, ${ }^{13}$ we have divided the registration method in two steps. In the first one only the structures with higher gradients are aligned, matching the patient skin, bones, gold fiducial markers placed in the prostate for localization purposes, and gas inside the bowels. The obtained deformation vector field is used to initialize the second step, where the lower gradients are aligned, and matching muscular and conjunctive structures.

\section{II.C. Evaluation}

To objectively evaluate and quantify the performance of the method the physician's manual contours have been chosen as our ground truth. Jaccard index (JI) ${ }^{21}$ and Dice similarity coefficient (DSC) ${ }^{22}$ are accepted as useful metrics for the quantification of the similarity between two sets. Nevertheless, since we intend to compare our results with the commented work of Gao et al., we use the overlap volume index (OVI) to quantify the differences between the automatic contours and the manual contours of the rectum. The OVI is defined as

$$
\text { OVI }=\text { Rectum }^{\text {manual }} \cap \text { Rectum }^{\text {automatic }} / \text { Rectum }^{\text {manual }} .
$$

Previous works have indicated that a DSC $>0.7$ shows a satisfactory overlapping between two volumes ${ }^{5,23,24}$ and the same value was used in the case of OVI. ${ }^{14}$

Furthermore, the evaluation method previously proposed by the authors ${ }^{25}$ is applied to the contours of the prostate to determine the influence of a better registration of the rectum in the automatic delineation of the prostate. This methodology allows the representation of the spatial distribution of the error around the reference prostate, showing the delineation variability with respect to the relative position on the prostate. ${ }^{5,25}$

\section{RESULTS AND DISCUSSION}

First of all, we are going to analyze the image database used in this article. As it can be seen in Table I, 26 images from 13 of our database present rectal filling volumes between 0.13 and $57.41 \mathrm{ml}$, with a mean value over $15 \mathrm{ml}$ and a standard deviation of almost $17 \mathrm{ml}$. If we studied the database used by Gao et $a l .,{ }^{14}$ the only work centered on distended rectums, 30 images from 15 patients have a mean volume value around $35 \mathrm{ml}$ and a standard deviation around $22 \mathrm{ml}$, with

TABLE I. Statistics for overlapped volume index using rigid registration, RMM, DRI, DRI using the manual segmentations, DRI with RMM in both images, and the proposed method (DRI with manual segmentation in the planning image and RMM in the daily image).

\begin{tabular}{|c|c|c|c|c|c|c|c|c|}
\hline Patient & Moving $\rightarrow$ fixed & Rectal fillings (ml) & Rigid alignment & RMM & DIR & DIR + Mask & $\mathrm{DIR}+\mathrm{RMM}$ & DIR mixed \\
\hline \multirow[t]{2}{*}{1} & $\mathrm{CT} 1 \rightarrow \mathrm{CT} 2$ & 11.68 & 0.72 & 0.97 & 0.85 & 0.96 & 0.91 & 0.93 \\
\hline & $\mathrm{CT} 2 \rightarrow \mathrm{CT} 1$ & 0.33 & 0.59 & 0.94 & 0.61 & 0.96 & 0.95 & 0.93 \\
\hline \multirow[t]{2}{*}{2} & $\mathrm{CT} 1 \rightarrow \mathrm{CT} 2$ & 4.92 & 0.48 & 0.93 & 0.8 & 0.96 & 0.9 & 0.85 \\
\hline & $\mathrm{CT} 2 \rightarrow \mathrm{CT} 1$ & 1.46 & 0.64 & 0.85 & 0.94 & 0.94 & 0.9 & 0.86 \\
\hline \multirow[t]{2}{*}{3} & $\mathrm{CT} 1 \rightarrow \mathrm{CT} 2$ & 0.13 & 0.51 & 0.92 & 0.72 & 0.96 & 0.87 & 0.79 \\
\hline & $\mathrm{CT} 2 \rightarrow \mathrm{CT} 1$ & 1.41 & 0.59 & 0.85 & 0.86 & 0.92 & 0.89 & 0.8 \\
\hline \multirow[t]{2}{*}{4} & $\mathrm{CT} 1 \rightarrow \mathrm{CT} 2$ & 0.13 & 0.75 & 0.92 & 0.78 & 0.97 & 0.92 & 0.83 \\
\hline & $\mathrm{CT} 2 \rightarrow \mathrm{CT} 1$ & 5.05 & 0.64 & 0.95 & 0.68 & 0.97 & 0.92 & 0.87 \\
\hline \multirow[t]{2}{*}{5} & $\mathrm{CT} 1 \rightarrow \mathrm{CT} 2$ & 19.9 & 0.68 & 0.91 & 0.83 & 0.97 & 0.9 & 0.91 \\
\hline & $\mathrm{CT} 2 \rightarrow \mathrm{CT} 1$ & 13.92 & 0.69 & 0.91 & 0.79 & 0.88 & 0.84 & 0.81 \\
\hline \multirow[t]{2}{*}{6} & $\mathrm{CT} 1 \rightarrow \mathrm{CT} 2$ & 55.13 & 0.53 & 0.88 & 0.76 & 0.94 & 0.91 & 0.9 \\
\hline & $\mathrm{CT} 2 \rightarrow \mathrm{CT} 1$ & 57.41 & 0.52 & 0.93 & 0.76 & 0.95 & 0.89 & 0.94 \\
\hline \multirow[t]{2}{*}{7} & $\mathrm{CT} 1 \rightarrow \mathrm{CT} 2$ & 36.05 & 0.72 & 0.85 & 0.9 & 0.98 & 0.84 & 0.96 \\
\hline & $\mathrm{CT} 2 \rightarrow \mathrm{CT} 1$ & 23.32 & 0.86 & 0.78 & 0.86 & 0.96 & 0.94 & 0.96 \\
\hline \multirow[t]{2}{*}{8} & $\mathrm{CT} 1 \rightarrow \mathrm{CT} 2$ & 31.58 & 0.44 & 0.75 & 0.64 & 0.98 & 0.88 & 0.98 \\
\hline & $\mathrm{CT} 2 \rightarrow \mathrm{CT} 1$ & 12.14 & 0.79 & 0.77 & 0.83 & 0.96 & 0.89 & 0.96 \\
\hline \multirow[t]{2}{*}{9} & $\mathrm{CT} 1 \rightarrow \mathrm{CT} 2$ & 0.5 & 0.73 & 0.75 & 0.9 & 0.97 & 0.97 & 0.94 \\
\hline & $\mathrm{CT} 2 \rightarrow \mathrm{CT} 1$ & 12.22 & 0.37 & 0.92 & 0.86 & 0.99 & 0.8 & 0.98 \\
\hline \multirow[t]{2}{*}{10} & $\mathrm{CT} 1 \rightarrow \mathrm{CT} 2$ & 0.5 & 0.7 & 0.77 & 0.78 & 0.98 & 0.97 & 0.98 \\
\hline & $\mathrm{CT} 2 \rightarrow \mathrm{CT} 1$ & 31.48 & 0.17 & 0.92 & 0.35 & 0.98 & 0.78 & 0.96 \\
\hline \multirow[t]{2}{*}{11} & $\mathrm{CT} 1 \rightarrow \mathrm{CT} 2$ & 18.48 & 0.62 & 0.9 & 0.9 & 0.99 & 0.92 & 0.96 \\
\hline & $\mathrm{CT} 2 \rightarrow \mathrm{CT} 1$ & 1.13 & 0.64 & 0.65 & 0.47 & 0.74 & 0.65 & 0.71 \\
\hline \multirow[t]{2}{*}{12} & $\mathrm{CT} 1 \rightarrow \mathrm{CT} 2$ & 35.75 & 0.31 & 0.77 & 0.51 & 0.94 & 0.81 & 0.88 \\
\hline & $\mathrm{CT} 2 \rightarrow \mathrm{CT} 1$ & 0.6 & 0.64 & 0.85 & 0.76 & 0.97 & 0.64 & 0.92 \\
\hline \multirow[t]{2}{*}{13} & $\mathrm{CT} 1 \rightarrow \mathrm{CT} 2$ & 14.99 & 0.6 & 0.92 & 0.81 & 0.97 & 0.98 & 0.97 \\
\hline & $\mathrm{CT} 2 \rightarrow \mathrm{CT} 1$ & 3.99 & 0.68 & 0.97 & 0.86 & 0.92 & 0.76 & 0.76 \\
\hline Mean & & 15.16 & 0.6 & 0.87 & 0.76 & 0.95 & 0.87 & 0.89 \\
\hline Standard deviation & & 16.88 & 0.15 & 0.09 & 0.14 & 0.05 & 0.09 & 0.08 \\
\hline
\end{tabular}




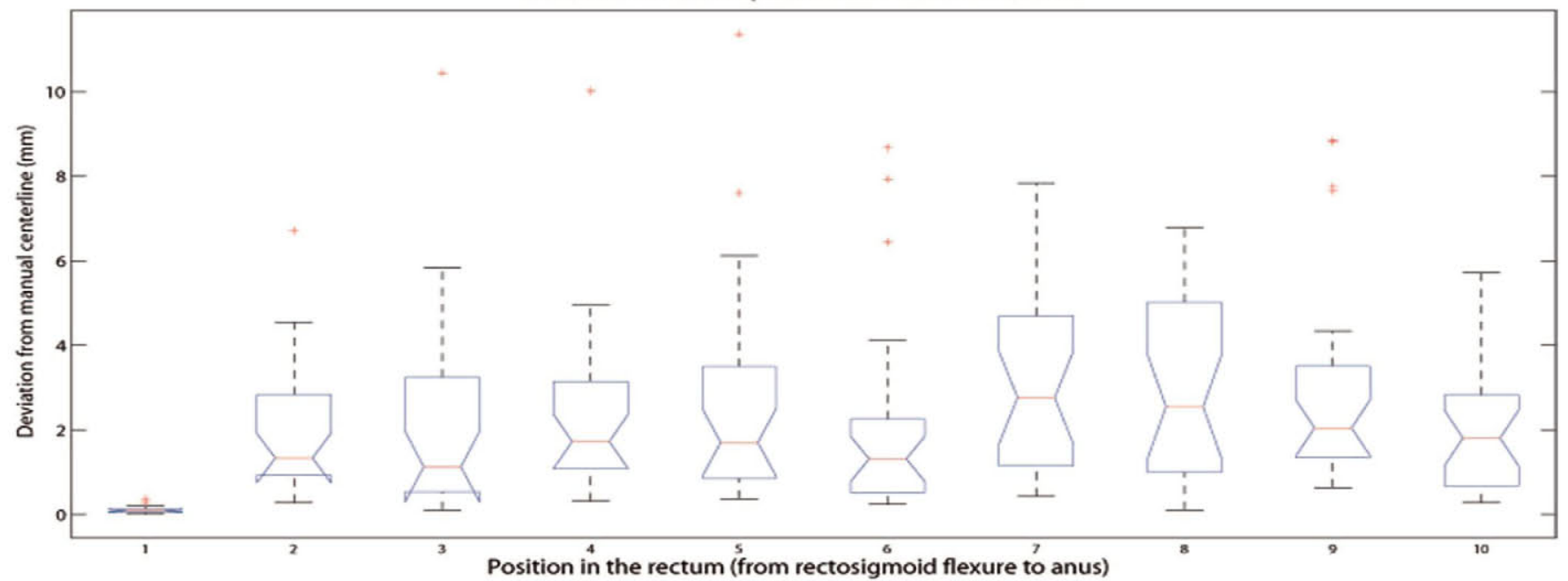

FIG. 5. The deviation of the semiautomatic estimation of the centerline from the manual centerline varies depending on the location of the slice. The slices closer to the rectosigmoid flexure have lower values of deviation than the slices in the middle. The mean value of deviation is always between 0 and $3 \mathrm{~mm}$.

4 outliers below $13 \mathrm{ml}$ and 3 outliers over $67 \mathrm{ml}$. Although our database itself and the rectal filling volumes are slightly smaller, the authors consider they are comparable for validation purposes.

There are present in the image database the 4 different combinations between the planning image and the daily image: (1) patients with an image with an almost empty rectum and the other image with a big volume of internal fillings (left column of Fig. 4); (2) the opposite, if we change the planning image and the daily image for registration purposes; (3) patients with images with large rectal volumes due to gas or feces (middle column in Fig. 4); and (4) patients with images with almost no rectal fillings in both cases (right column in Fig. 4). Thus, the evaluation of the method takes into account all the possibilities during the clinical treatment.

The evaluation of the RMM is divided into two different steps: the evaluation of the centerline estimation and the evaluation of the final segmentation. Good centerline estimation is basic for a correct segmentation, since the RMM method starts from the centerline of the rectum. The deviation between the manual centerline and the estimated centerline of 26 images is presented in Fig. 5 using an ANOVA representation. Since the centerlines have not the same length, all of them have been interpolated into 10 slices equally distributed between the rectosigmoid flexure (column 1) and the anus (column 10). As it can be seen in Fig. 5, the mean deviation from the manual centerline changes depending on the position of the slice, presenting lower values when the slices are closer to the manual seeds. Nevertheless, the mean deviation is always between 0 and $3 \mathrm{~mm}$, and the centerline estimation is inside the manual segmentation of the rectum in the whole image for 25 of 26 cases.

The case where the estimation fails presents a special characteristic: the strangulation of the rectum in the rectosigmoid flexure makes the estimation fail in the slices closer to the seed. Two additional landmarks in the slices where the strangulation appears help solving this problem.
An example of the whole RMM process performance is depicted in Fig. 6: Fig. 6(a) shows the original CT slice, centered in the rectum's center of mass, and the final contour computed. Figure 6 (b) presents the searching image, where can be easily distinguished the conjunctive-like tissues in white, the muscular tissues in gray, and the rectal fillings in black. Finally, Fig. 6(c) represents the polar-log transformed image, where the rectum wall appears represented as an almosthorizontal line evolving muscular tissue and rectal fillings.

To compare the effectiveness of the RMM, we have performed DIR to a pair of prostate CT images with and without using the RMM method. The example is represented in Fig. 7. The planning CT has a small empty rectum while the

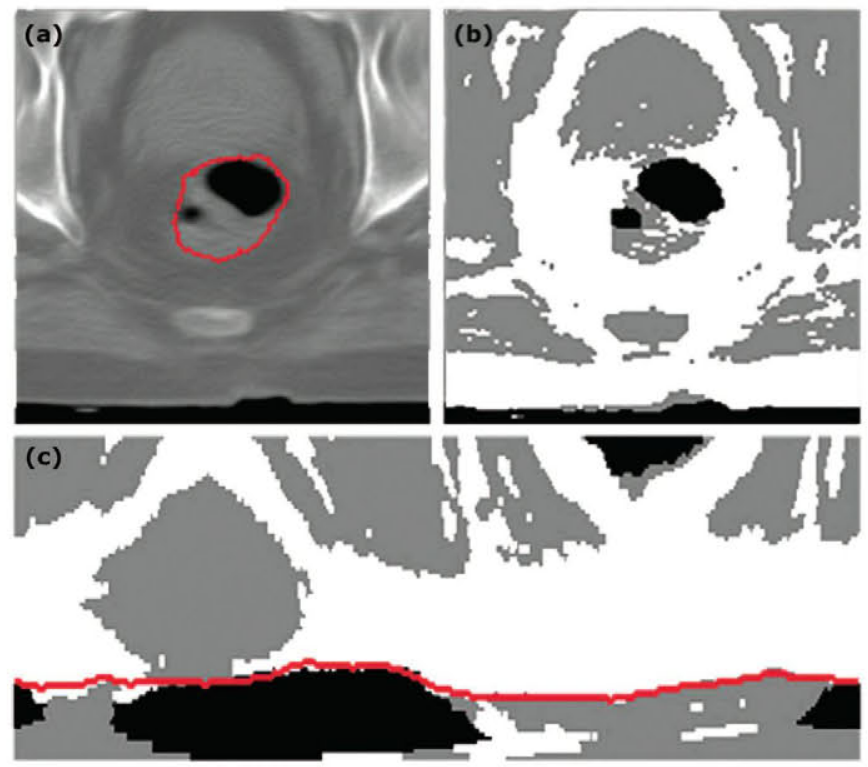

FIG. 6. (a) Original CT image showing a distended rectum and the prostate. (b) Searching image representing the conjunctive-like tissue (white), the muscular tissue (gray), and rectal fillings (black). (c) Polar-log transformed image showing the rectum wall as an almost-horizontal line. 
Planning CT
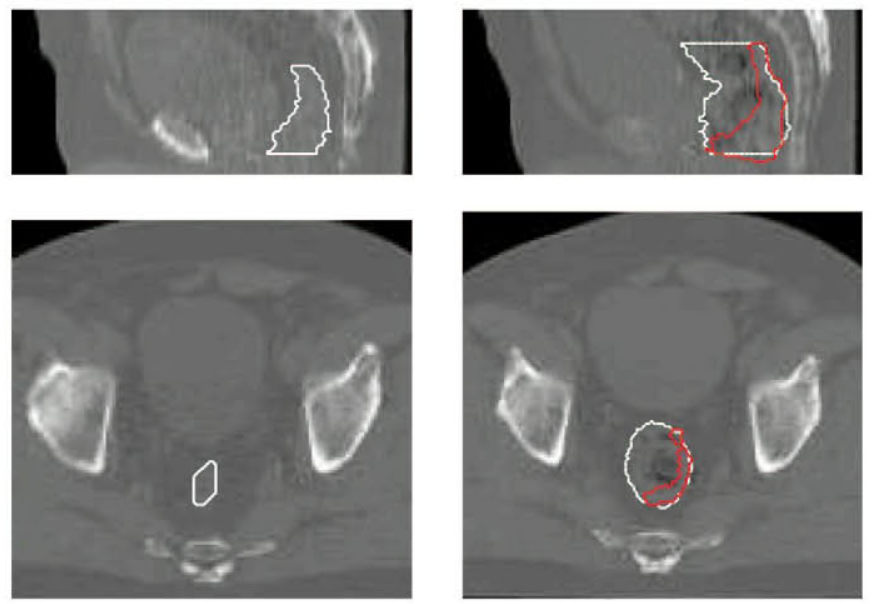

FIG. 7. The CT images of one prostate cancer patient with the registered contours overlaid. (Left) Planning CT without rectal fillings in sagittal and axial slices, and the manual contours overlaid. (Right) The corresponding slices of the daily CT with the deformed contours overlaid. The bright and dark contours represent the segmentation results with (bright) and without (dark) the RMM.

daily CT has a large amount of rectal fillings that significantly distend the rectum. Two representative sagittal and axial slices are shown in order to illustrate the results. The manual contour (bright) is overlaid in the planning image, while two set of contours are overlaid in the daily image: the deformed contours without using RMM (shown in dark) and the deformed contours using RMM (shown in bright). As it can be seen in the figure, the deformed contours without using RMM clearly did not match with the rectum due to the lack of one-to-one correspondence.

RMM may fail if the intensity and/or the shape condition are not met. For example, the intensity condition may be affected if a high amount of solid feces inside the rectum is labeled as conjunctive-like tissue [see Fig. 8(a)]. However, the use of radiological contrast in the bladder should not affect negatively. The shape condition may be compromised if strangulation appears on the rectum, or if the rectum and superior regions of the bowels appear in the same slice stuck together [see Fig. 8(b)]. In these last cases, additional landmarks will increase the robustness of the method. On the contrary, if the intensity condition fails, there is not an obvious solution.
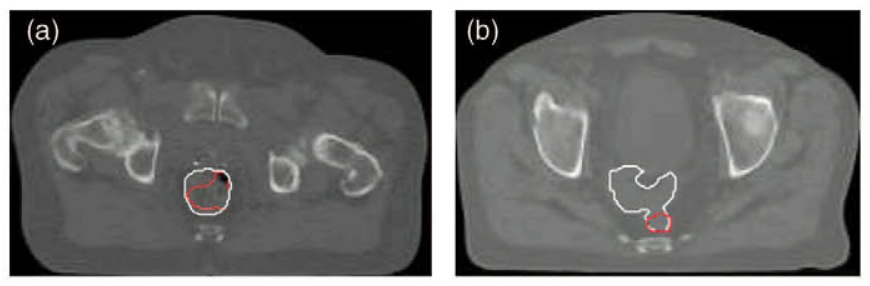

FIG. 8. The RMM method may fail if the intensity or the shape conditions are not met. The manual contours (white) and the RMM contours (red) are overlaid. (a) The RMM fails in the segmentation due to an incorrect labeling of the rectal fillings. (b) The strangulation of the rectum did not meet the condition of a tubular shape, and the RMM does not model the complex contour.
Although the purpose of RMM is not to obtain an accurate segmentation of the rectum, but a volume that encompasses it, the study of the OVI values of the obtained contours and their respective manual delineations offers a basis for following analyses. It is important to highlight that low similarities between the mask and the actual rectum affect the performance of posterior processes. As can be seen in Table I, the RMM masks and the manual delineations of the rectum have a mean value of $\mathrm{OVI}_{\mathrm{RMM}}=0.87$ and a minimum value of $\mathrm{OVI}_{\mathrm{RMM}}=0.65$, which is a good similarity value, basic for obtaining good results after the DIR.

The initial hypothesis of the method is that the DIR performance will be positively affected if the rectum is segmented and masked in both images. The manual segmentation of the images has been used to evaluate this statement. The seventh column of Table I shows the results of the combination of the DIR and the manual segmentations, presenting a mean value of OVI $=0.95$, which is almost a perfect matching.

The images with rigid alignment have a mean overlap value of OVI $=0.60$, below the similarity threshold commented above. The application of the DIR algorithm increases this value to $O V I_{\text {DIR }}=0.76$, which is better than the commented threshold of OVI $=0.7$, but gives us a big margin for improvement until the value obtained using the manual masks. The application of the RMM to both images lead to a mean value of $\mathrm{OVI}_{\mathrm{RMM}}=0.87$, with two cases where the similarity value is below 0.7 . Nevertheless, the use of the manual segmentation in the planning image (always available) and the RMM mask in the daily image leads to a mean value of $\mathrm{OVI}_{\mathrm{MIX}}=0.89$, and all the cases are above the similarity threshold. This improvement is related to the accuracy of the manual segmentations compared to the masks obtained using the RMM. Only in one case the results of the DIR alone are better than the use of the proposed method, but no conclusion can be extracted from this single case.

If we compare the obtained results with the paper of Gao et al. ${ }^{14}$ we have to take into account that the differences may be due to both the different preprocess of the image and/or the different IR algorithm. In Gao et al. the application of the DIR algorithm did not suppose any improvement against the rigid alignment, offering both a value of OVI $=0.51$. The introduction of the image intensity modification (IMM) method, that creates artificial gas bubbles in the planning image when necessary, supposed an improvement of 0.20 , reaching a value of $\mathrm{OVI}_{\mathrm{IMM}}=0.71$, slightly better than the similarity threshold, but in 10 cases the results were below 0.70 .

On the other hand, the results presented in this article show a relative improvement of 0.13 in the overlapping volume, but higher absolute values. The DIR results without preprocessing are much better than the results presented by Gao et al. This may have its origin in the different database and/or in the DIR performance. The actual influence of the preprocessing step (the IMM method in the case of Gao et al., and the RMM in this case) in the final results can be evaluated using the same DIR algorithm in both cases, but this task is not part of this research.

Moreover, the planning images used in Ref. 14 are pelvic images without any kind of rectal fillings, since the patients 

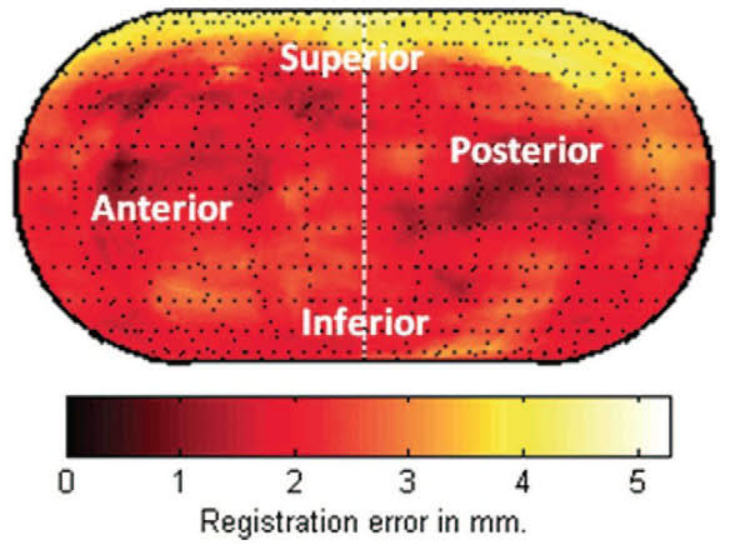

FIG. 9. Map of the registration error over the prostate. The largest error is located in the superior region, near the bladder.

have to overcome an enema to empty the rectum. In our case, the planning image does not need any special interaction and the patients do not need to suffer an enema.

Measuring the influence of a correct rectum registration over the prostate registration is a difficult task. A quantitative evaluation method that allows the representation of the delineation error distribution around the reference prostate has been used. The deviation of the registered contours from the reference contours can be visualized using the centroid of the planning image prostate as a reference point. Thus, we consider the deviation of the contour in a specific direction as the minimum distance between the intersection points between the direction vector and the prostate surface. This procedure produces a distance for each radial direction, which can be plotted on the surface of a sphere, thus obtaining a radial distance map. Since the patients are all scanned in the same orientation, average maps can be computed.

Figure 9 shows the mean radial distance at each point for the cases analyzed in this study. It can be noticed that the largest variation is found in the superior direction, which is consistent with the reported difficulty of detecting the prostate-bladder interface. ${ }^{5}$ Also, the inferior direction has some high values, due to the inconsistency of the apex delineation. The posterior middle region, the one more influenced by the rectum deformations, has the lowest values of variability.

\section{CONCLUSIONS}

The authors have proposed a RMM that modifies the intensity of the rectum and its content (gas or feces) to improve the performance of intensity-based DIR methods in prostate cancer ART. The application of the RMM method in the daily image and the manual segmentations in the planning image during prostate cancer treatments increases the performance of the registration in presence of rectal fillings. We have evaluated the proposed method in 13 patient cases and 26 pairs of registrations, obtaining very good agreement with a physician's manual contours. Moreover, the results show that a more accurate delineation of the rectum supposes a small im- provement in the prostate delineation, located in the posterior side of the prostate.

\section{ACKNOWLEDGMENTS}

The authors would like to thank Dr. Almudena Zapatero Laborda, from the Oncologic Radiotherapy Department of Hospital Universitario de La Princesa, for providing the manual contours of the patients.

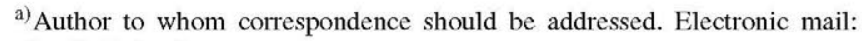
brvila@gbt.tfo.upm.es

${ }^{1}$ D. Yan and D. Lockman, "Organ/patient geometric variation in external beam radiotherapy and its effects," Med. Phys. 28(4), 593-602 (2001).

${ }^{2}$ E. Huang, L. Dong, A. Chandra, D. A. Kuban, I. I. Rosen, A. Evans, and A. Pollack, "Intrafraction prostate motion during IMRT for prostate cancer,” Int. J. Radiat. Oncol., Biol., Phys. 53(2), 261-268 (2002).

${ }^{3}$ International Commission on Radiation Units and Measurements (ICRU), "Prescribing, recording and reporting photom beam therapy," Report No. 50 (1993).

${ }^{4}$ D. Yan, F. Vicini, J. Wong, and A. A. Martinez, "Adaptive radiation therapy,” Phys. Med. Biol. 42(1), 123-132 (1997).

${ }^{5}$ M. Foskey, B. Davis, L. Goyal, S. Chang, E. Chaney, N. Strehl, S. Tomei, J. Rosenman, and S. Joshi, "Large deformation three-dimensional image registration in image-guided radiation therapy," Phys. Med. Biol. 50, 58695892 (2005).

${ }^{6}$ D. C. Collier, S. S. C. Burnett, M. Amin, S. Bilton, C. Brooks, A. Ryan, D. Roniger, D. Tran, and G. Starkschall, "Assessment of consistency in contouring of normal-tissue anatomic structures," J. Appl. Clin. Med. Phys. 4(1), 417-424 (2003).

${ }^{7}$ W. R. Crum, T. Hartkens, and D. L. G. Hill, "Non-rigid image registration: Theory and practice," Br. J. Radiol. 77, 140-153 (2004).

${ }^{8}$ P. J. Keall, S. Joshi, S. S. Vedam, J. V. Siebers, V. R. Vini, and R. Mohan, "Four-dimensional radiotherapy planning for DMLC-based respiratory motion tracking," Med. Phys. 32, 942-951 (2005).

${ }^{9}$ W. Lu, G. H. Olivera, Q. Chen, M. L. Chen, and K. J. Ruchala, “Automatic re-contouring in 4D radiotherapy," Phys. Med. Biol. 51, 1077-1099 (2006).

${ }^{10}$ C. Wu, R. Jeraj, G. H. Olivera, and T. R. Mackie, "Re-optimization in adaptive radiotherapy," Phys. Med. Biol. 47, 3181-3195 (2004).

${ }^{11}$ G. E. Christensen, B. Carlson, K. S. C. Chao, P. Yin, P. W. Grigsby, K. Nguyen, J. F. Dempsey, F. A. Lerma, K. T. Bae, M. W. Vannier, and J. F. Williamson, "Image-based dose planning of intracavitary brachytherapy: Registration of serial-imaging studies using deformable anatomic templates,” Int. J. Radiat. Oncol., Biol., Phys. 51, 227-243 (2001).

${ }^{12}$ H. Wang, L. Dong, J. O'Daniel, R. Mohan, A. S. Garden, K. K. Ang, D. A. Kuban, M. Bonnen, J. Y. Chang, and R. Cheung, "Validation of an accelerated 'demons' algorithm for deformable image registration in radiation therapy," Phys. Med. Biol. 50(12), 2887-2905 (2005).

${ }^{13}$ S. S. Samant, J. Xia, P. Muyan-Özçelik, and J. D. Owens, "High performance computing for deformable image registration: Towards a new paradigm in adaptive radiotherapy," Med. Phys. 35(8), 3546-3553 (2008).

${ }^{14}$ S. Gao, L. Zhang, R. De Crevoisier, D. D. Kuban, R. Mohan, and L. Dong, "A deformable image registration method to handle distended rectums in prostate cancer radiotherapy," Med. Phys. 33(9), 3304-3312 (2006).

${ }^{15}$ G. T. Herman, Fundamentals of Computerized Tomography: Image Reconstruction from Projection, 2nd ed. (Springer, 2009).

${ }^{16} \mathrm{~J}$. Weickert and H. Scharr, "A scheme for coherence-enhancing diffusion filtering with optimized rotation invariance," J. Visual Commun. Image Representation 13(1-2), 103-118 (2002).

${ }^{17}$ W. Lu, G. H. Olivera, Q. Chen, K. J. Ruchala, J. Haimerl, S. L. Meeks, K. M. Langen, and P. A. Kupelian, "Deformable registration of the planning image (kVCT) and the daily images (MVCT) for adaptive radiation therapy," Phys. Med. Biol. 51, 4357-4374 (2006).

${ }^{18}$ International Commission on Radiation Units and Measurements (ICRU), "Prescribing, recording and reporting photom beam therapy," Report No. 62 (1999) (Supplement to ICRU Report No. 50).

${ }^{19}$ M. A. Luengo-Oroz and J. Angulo, "Cyclic mathematical morphology in polar-logarithmic representation," IEEE Trans. Image Process. 8(5), 10901096 (2009). 
${ }^{20}$ T. Vercauteren, X. Pennec, A. Perchant, and N. Ayache, "Non-parametric diffeomorphic image registration with the Demons algorithm," Med. Image Comput. Computer Assist. Interven. 10(2), 319-326 (2007).

${ }^{21} \mathrm{P}$. Jaccard, "Étude comparative de la distribution florale dans une portion des Alpes et des Jura," Bull. Soc. Vaudoise Sci. Nat. 37, 547-579 (1901).

${ }^{22}$ L. R. Dice, "Measures of the amount of ecologic association between species," Ecology 26(3), 297-302 (1945).

${ }^{23}$ D. Goldberg-Zimring, I. Talos, J. G. Bhagwat, S. J. Haker, P. M. Black, and K. H. Zou, "Statistical validation of brain tumor shape approximation via spherical harmonics for image-guided neurosurgery," Acad. Radiol. 12, 459-466 (2005).

${ }^{24}$ K. H. Zou, S. K. Warfield, A. Baharatha, C. Tempany, M. R. Kaus, S. J. Haker, W. M. Wells, F. A. Jolesz, and R. Kikinis, "Statistical validation on image segmentation quality based on a spatial overlap index," Acad. Radiol. 11, 178-189 (2004).

${ }^{25}$ B. Rodriguez-Vila, F. Gaya, F. Garcia-Vicente, and E. J. Gomez, "Threedimensional quantitative evaluation method of non-rigid registration algorithms for adaptive radiotherapy," Med. Phys. 37(3), 1137-1145 (2010). 